\title{
Phylogenomic analysis of Pseudomonas nitroreducens strains FY43 and FY47
}

\author{
Xue Li Tana, Wei Yee Wee ${ }^{b}$, Boon Chin Tan ${ }^{a}$, Chee How Teo ${ }^{a^{*}}$ \\ ${ }^{a}$ Centre for Research in Biotechnology for Agriculture (CEBAR), Universiti Malaya, 50603 Kuala Lumpur, Malaysia \\ ${ }^{b}$ School of Science, Monash University Malaysia, 47500 Subang Jaya, Malaysia
}

Received 22nd July 2021 / Accepted 6th December 2021

\begin{abstract}
Proper identification of strain is essential in understanding the ecology of a bacteria species. The classification of Pseudomonas nitroreducens is still being questioned and revised until now. The novel $P$. nitroreducens strains FY43 and FY47 used in this study have been reported to show a high level of tolerance to glyphosate. In this study, next-generation sequencing (NGS) and whole genome analysis were used to clarify the delineation of the species. Whole genome analysis showed that P. nitroreducens strains FY43 and FY47 shared high homology to five reference genomes of P. nitroreducens: strain B, Aramco J, NBRC 12694, DF05, and TX01. Phylogenomic and phylogenetic analysis (average nucleotide identity based on BLAST (ANIb), genome-to-genome distance (GGDC) analysis) showed that both P. nitroreducens strains FY43 and FY47 are Pseudomonas nitroreducens members. However, strains DF05 and TX01 were not correctly assigned at the species level for all the analyses. The P. nitroreducens strain DF05 and TX01 should be further investigated for their classification as the correct species classification is the prerequisite for future diversity studies.
\end{abstract}

Keywords: ANIb, GGDC, MLSA, Next-generation Sequencing, Pseudomonas nitroreducens

\section{INTRODUCTION}

Pseudomonas is a genus of rod-shaped and gramnegative bacteria (Euzéby, 1997). There are 216 species of Pseudomonas sp., including 18 subspecies, which have been recorded in the List of Prokaryotic Names with Standing in Nomenclature (Parte, 2014). Pseudomonas sp. can survive actively in water, soil and plants.

Identification and classification of Pseudomonas strains is a necessary step to understand the ecology of this genus. In the early years, Pseudomonas sp. was classified based on their phenotypes, such as nutritional screening (Stanier et al., 1966; Novik et al., 2015). In 1984, the taxonomy of the Pseudomonas genus was revised when using molecular techniques, such as DNADNA hybridization (DDH) and rRNA-DNA hybridization (Gomila et al., 2015). Gomila et al.
(2015) found that only 31 out of 96 previously approved Psendomonas sp. were considered as true species in the list of Pseudomonas sp. However, $\mathrm{DDH}$ values may not be reproducible as it is a laboratory-based technique, which may vary depending on the experimental settings and laboratory methods employed (Colston et al., 2014). Moreover, DDH requires a large amount of high-quality DNA, which could be labor intensive and time-consuming (Goris et al., 2007). The data generated from the DDH technique cannot be archived and shared between laboratories (Colston et al., 2014). Therefore, the DDH data might not be suitable for new species description (Goris et al., 2007; Colston et al., 2014).

Currently, 16S rRNA gene sequencing is commonly used for bacterial classification.

*Author for correspondence: Chee How Teo, Centre for Research in Biotechnology for Agriculture (CEBAR), Universiti Malaya, 50603 Kuala Lumpur, Malaysia. Email - cheehow.teo@um.edu.my 
However, this method is often questioned due to insufficient resolution for specific species identification (Mulet et al., 2010). To enhance the phylogenetic resolution, a few reference genes are often used as phylogenetic molecular markers. This is because these reference genes are highly conserved and have a high degree of sequence divergence, especially at the genus level (Glaeser \& Kämpfer, 2015). However, a single gene-based sequence does not reflect the accurate phylogenetic relationship. Hence, a multiple genebased phylogenetic analysis has been developed to eliminate the bias created from the single genebased sequence analysis. Multilocus sequence analysis (MLSA) was introduced by Maiden et al. (1998). This method involves concatenating several reference gene fragments to build a phylogenetic tree based on fragment similarities. Four reference genes, namely $16 \mathrm{~S}$ rRNA, rpoB, $r p o \mathrm{D}$, and $g y r \mathrm{~B}$, have been proven reliable in strain classification and species delineation for Pseudomonas sp. (Mulet et al., 2013; Garrido-Sanz et al., 2017).

Next-generation sequencing (NGS) is a costeffective sequencing method for comparative genomic study. It can produce high-quality whole genome sequences, which is useful for taxonomic relationships and evolutionary studies (Chain $e t$ al., 2009; Gomila et al., 2015). With NGS, new taxa could be discovered, and readily built genomic data could be shared among researchers (Colston et al., 2014). Two bioinformatics tools, namely genome-to-genome distance calculator (GGDC) and average nucleotide identity (ANI), have been developed for NGS analysis. GGDC is a substitution for $\mathrm{DDH}$, whereby it gives values that are comparable to experimental-based DDH values (Meier-Kolthoff et al., 2013). ANI calculates the mean of similarity values between two homologous genomic sequences. It is now a new standard for microbial species classification (Konstantinidis et al., 2006). Previous studies demonstrated that species identification was improved by combining MLSA or analysis based on common genes in the genus with the ANI tool (Chan et al., 2012; Tarazona et al., 2014). To date, many Pseudomonas strains have been studied and classified, but the correct taxonomic position remains uncertain. For instance, Pseudomonas oryzibabitans was omitted from the classification of P. psychrophila when analyzed using the DDH method (Yumoto et al., 2001). However, the recent re-classification of both species was conducted using phylogenetic analysis, MLSA (four housekeeping genes, 16S rRNA, gyr $\mathrm{B}, r p o \mathrm{~B}$ and $r p o \mathrm{D}$ were included), and phylogenomic analysis (ANIb and GGDC) (Lalucat et al., 2020). The analysis showed that both species are the same genomospecies (Lalucat et al., 2020).

Mulet et al. (2013) re-classified Pseudomonas putida strains based on gene sequences of $16 \mathrm{~S}$ rRNA, gyrB, and rpoD. The authors found that some strains previously classified as Pseudomonas putida were phylogenetically affiliated with other closest relatives in the same genus (Mulet et al., 2013). In total 29 genomes designated as Pseudomonas fluorescens may be misclassified as they are also found in 20 different genomic clusters (Jun et al., 2016). Therefore, a thorough taxonomic analysis is needed to assist comparative genomic analysis to avoid confusion in the identification and classification of Pseudomonas nitroreducens.

In this study, we generated high-quality draft genome sequences of Pseudomonas nitroreducens strains FY43 and FY47 using various genome assemblers. Furthermore, together with $P$. nitroredunces in this study and previously reported strains, we re-classified the $P$. nitroreducens species through phylogenomic approaches.

\section{MATERIALS AND METHODS}

\section{Whole genome sequencing and assembly for Pseudomonas nitroreducens strains FY43 and FY47}

Pseudomonas nitroreducens strains previously reported by Looi (2016) and Tan et al. (2020) were used in this study. The genomic DNA of FY43 and FY47 isolates were extracted using EasyPure ${ }^{\circledR}$ Bacteria Genomic DNA Kit (TransGen Biotech, China) according to the manufacturer's protocol. The extracted genomic DNA was sent for whole genome sequencing using NGS service provided by Apical Scientific Sdn. Bhd. and Next Gene Scientific Sdn. Bhd. The NGS libraries were prepared using VAHTS Universal DNA library prep kit for Illumina (Vazyme Biotech, China). Genome sequencing was performed using a high-throughput Illumina 
HiSeq X Ten technology platform with a $150 \mathrm{bp}$ paired-end strategy. Sequencing reads were preprocessed by removing poor quality reads (e.g. Phred score $\leq 20$ ) and ambiguous base calls (Ns) using Cutadapt (version 1.9.1). The postprocessed clean reads were subjected to de novo genome assembly using different assembly software, such as SPAdes (Bankevich et al., 2012), ABySS (Simpson et al., 2009), and Velvet assembler (Zerbino \& Birney, 2008). The best assembly/scaffold was chosen based on N50 values, number of contig/scaffold, and assembly/scaffold size. The assembled genome of FY43 and FY47 was submitted to NCBI GenBank under BioProject ID PRJNA779109.

\section{Phylogenetic inferences}

A total of 8 Psendomonas strains, namely 5 Pseudomonas nitroreducens strains (NBRC12694, Aramco J, B, FY43 and FY47), 2 Pseudomonas sp. strains DF05 and TX1 (originally classified as $P$. nitroreducens), and Pseudomonas denitrificans ATCC13867 (as an outgroup), were used to construct a phylogenetic tree based on four reference genes from each strain, namely $16 \mathrm{~S}$ rRNA, $g y r \mathrm{~B}, r p o \mathrm{~B}$, and $r p o \mathrm{D}$. The nucleotide sequences of each reference gene were aligned using CLUSTAL_X (Thompson et al., 1997) and constructed using the Maximum Likelihood method with 500 bootstrap replicates. The gene sequences of the $16 \mathrm{~S} r \mathrm{RNA}, g y r \mathrm{~B}, r p o \mathrm{~B}$, and $r p o \mathrm{D}$ were then concatenated for the multilocus sequence analysis. The construction of the supermatrix-based tree was the same as the single gene-based tree (Gomila et al., 2015).

\section{Average nucleotide identity (ANI) and genome-to-genome distance (GGDC)}

The average nucleotide identity based on the BLAST algorithm (ANIb) was calculated between genome pairs for each strain using JSpecies (Richter \& Rosselló-Móra, 2009). The genomes (the query) were cut into 1,020 nucleotide fragments before calculating the ANIb value between the query genome and the reference genome. The ANIb represents a mean identity of all the BLASTN matches showing more than 30\% overall sequence identity over an alignable region of at least $70 \%$ coverage of their length. GGDC was calculated using the web service http://ggdc.dsmz.de (Meier-Kolthoff et al., 2013).

\section{RESULTS AND DISCUSSION}

\section{De novo genome assembly of Pseudomonas nitroreducens strains FY43 and FY47}

The genomes of Pseudomonas nitroreducens strains FY43 and FY47 were sequenced and assembled de novo. The draft genome sizes of strains FY43 and FY476 were 6,255,176 and 6,266,999 bp, respectively (Supplementary Data). The contig number of FY43 and FY47 were 68 and 74, respectively. The assembled genome sizes of both Pseudomonas nitroreducens strains FY43 and FY47 in this study were in accordance with the genome sizes of Pseudomonas reported by Thakur et al. (2016) and Molina-Mora et al. (2020). According to the de novo assembly results from the previous studies, the genome sizes of Psendomonas sp. were in the range of $5,098,132$ to $7,190,208 \mathrm{bp}$, whereas the contig numbers were in between 86 to 684 (Thakur et al., 2016; Molina-Mora et al., 2020). In this study, the results showed that ABySS assembly software (Table 1) generated the largest genome size, highest N50 values, and lowest contig number compared to SPAdes and Velvet assemblers. Hence, ABySS assembly software was selected for the subsequent analysis.

Table 1. De novo genome assembly of Pseudomonas nitroreducens strains FY43 and FY47 analyzed from ABySS assembly software.

\begin{tabular}{lcc}
\hline ABySS & FY43 & FY47 \\
\hline Genome Size & $6,255,176$ & $6,266,999$ \\
Contig No & 68 & 74 \\
N50 & 239,855 & 240,409 \\
$\begin{array}{l}\text { Minimum } \\
\text { contig }\end{array}$ & 204 & 204 \\
$\begin{array}{l}\text { Maximum } \\
\text { contig }\end{array}$ & 858,264 & 863,737 \\
\hline
\end{tabular}




\section{Reclassification of Pseudomonas nitroreducens species based on phylogenetic analysis}

Phylogenetic analysis was conducted to clarify the delineation of Pseudomonas nitroreducens species. In this study, the sequences of $16 \mathrm{~S} r \mathrm{RNA}, g y r \mathrm{~B}, r p o \mathrm{~B}$, and $r p o \mathrm{D}$ from each strain were evaluated using a phylogenetic tree. Based on the 16S rRNA, Pseudomonas nitroreducens strains FY43 and FY47 were grouped with other Pseudomonas nitroreducens species, except DF05, TX01, and Pseudomonas denitrificans ATCC13867 (Figure 1). The finding suggests that DF05 and TX01 might not belong to Pseudomonas nitroreducens species. To further confirm this finding, phylogenomic analysis and MLSA on other genes, namely $g y r \mathrm{~B}, r p o \mathrm{~B}$, and $r p o \mathrm{D}$, were performed. Interestingly, all trees revealed that $P$. nitroreducens strains FY43 and FY47 were grouped with $P$. nitroreducens strain B, Aramco J, and NBRC 12694, whereas Pseudomonas sp. strains DF05 and TX01 were clearly separated from other strains (Figures 1 to 4). Phylogenetic analysis using $r p o \mathrm{D}$ gene showed that Pseudomonas sp. DF05 and TX01 formed a subclade that separated them from the rest of Pseudomoans nitroreducens species (Figure 4). Although the $r p o \mathrm{D}$ gene only grouped Pseudomonas strains into 2 main clades, it is still suitable and widely adopted for genotyping Pseudomonas genera (Mulet et al., 2009). These findings showed that Pseudomonas sp. strains DF05 and TX01 might be misclassified as Pseudomonas nitroreducens.

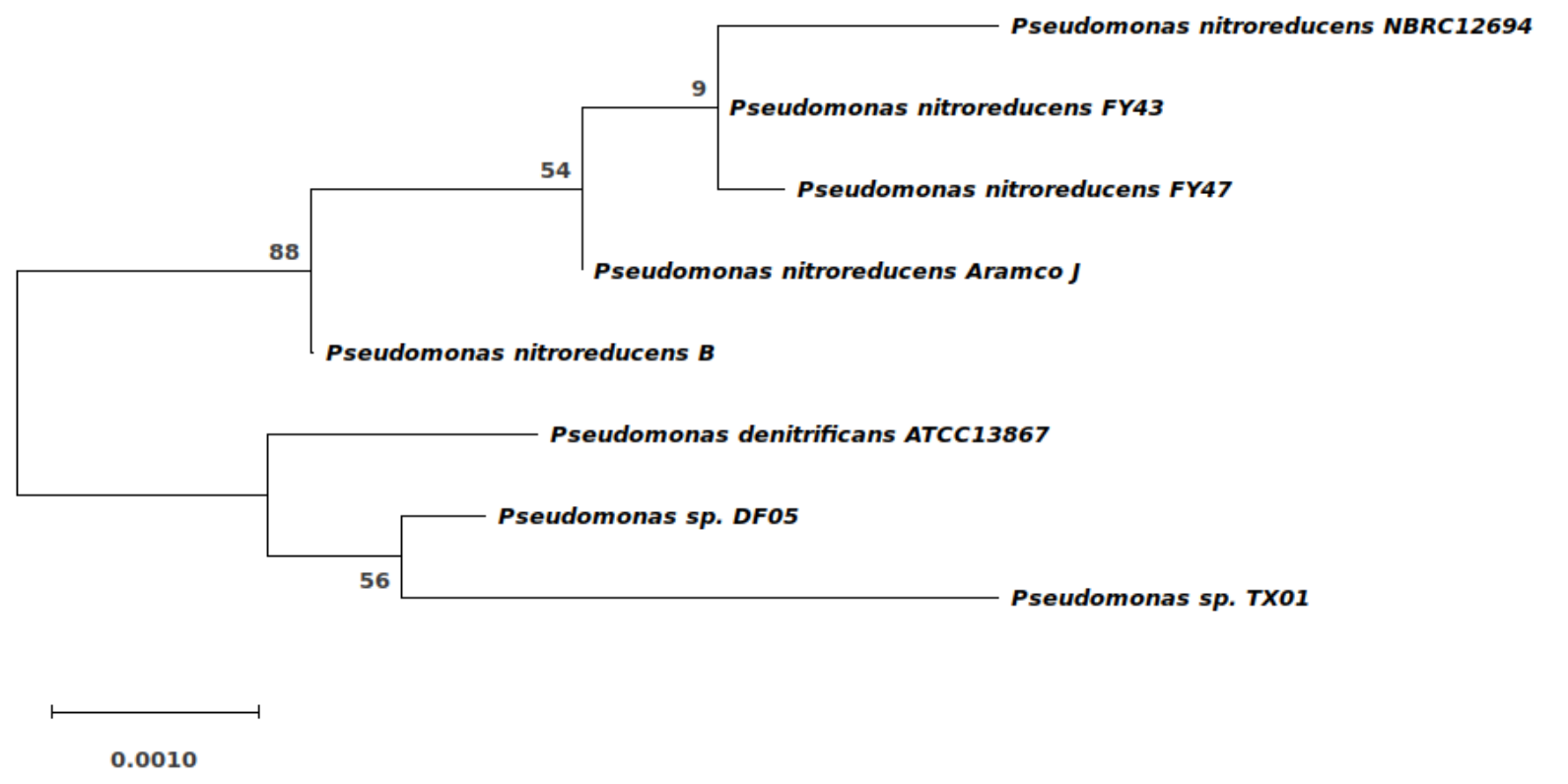

Figure 1. Phylogenetic tree of 8 Psendomonas strains based on 16S rRNA gene sequences. 


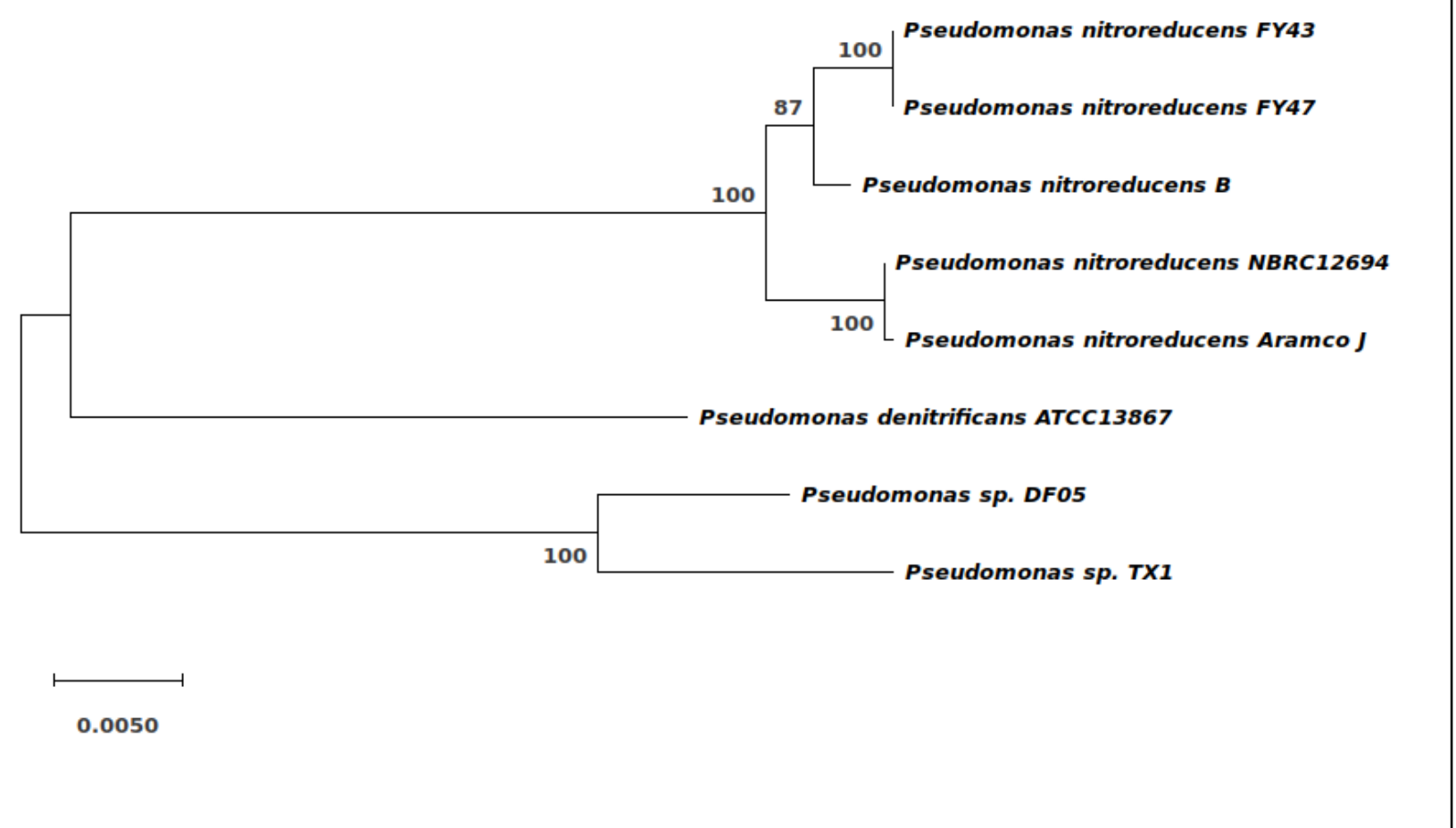

Figure 2. Phylogenetic tree of 8 Pseudomonas strains based on gyr Bene sequences.

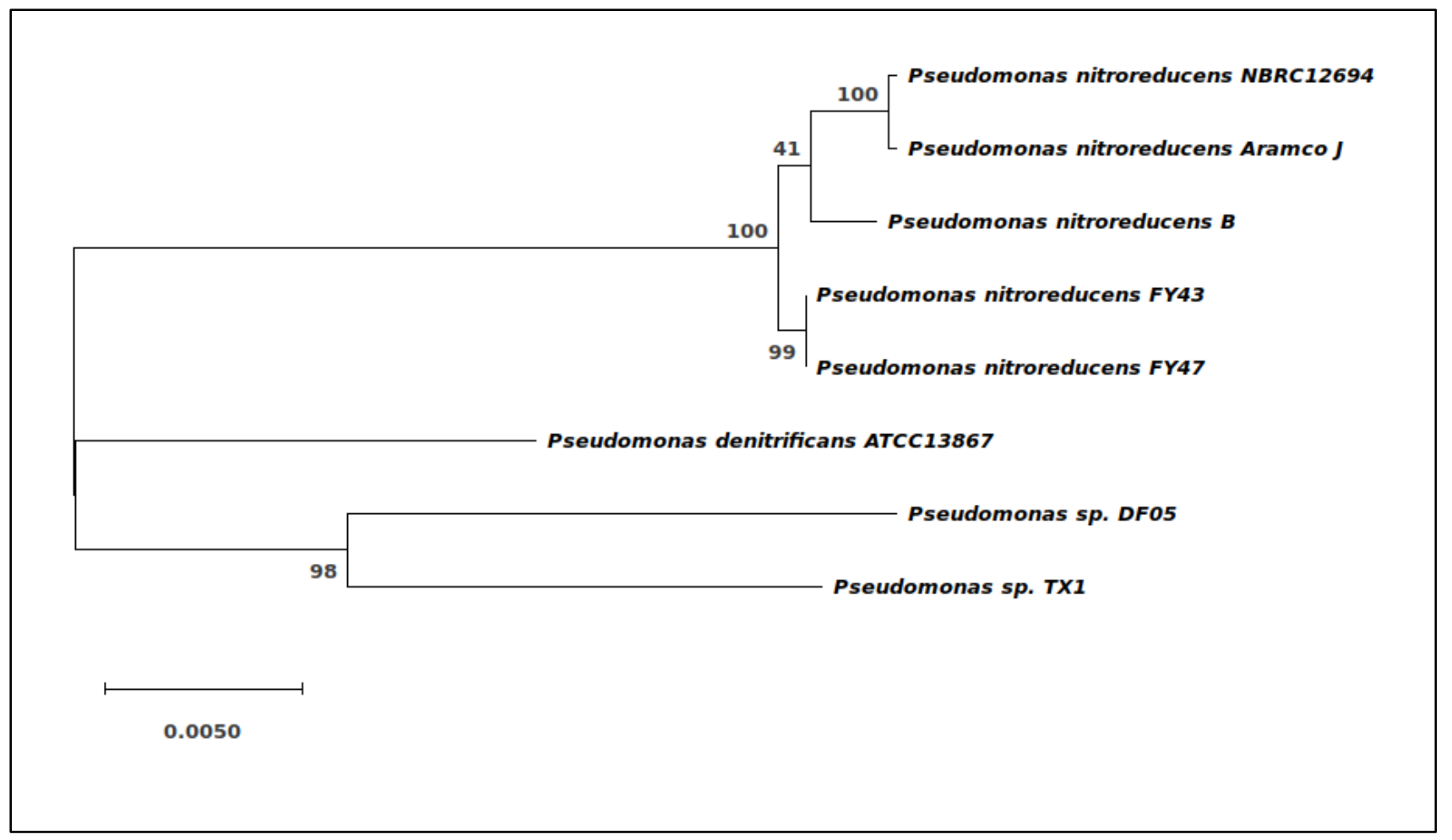

Figure 3. Phylogenetic tree of 8 Pseudomonas strains based on $r p o \mathrm{~B}$ gene sequences. 


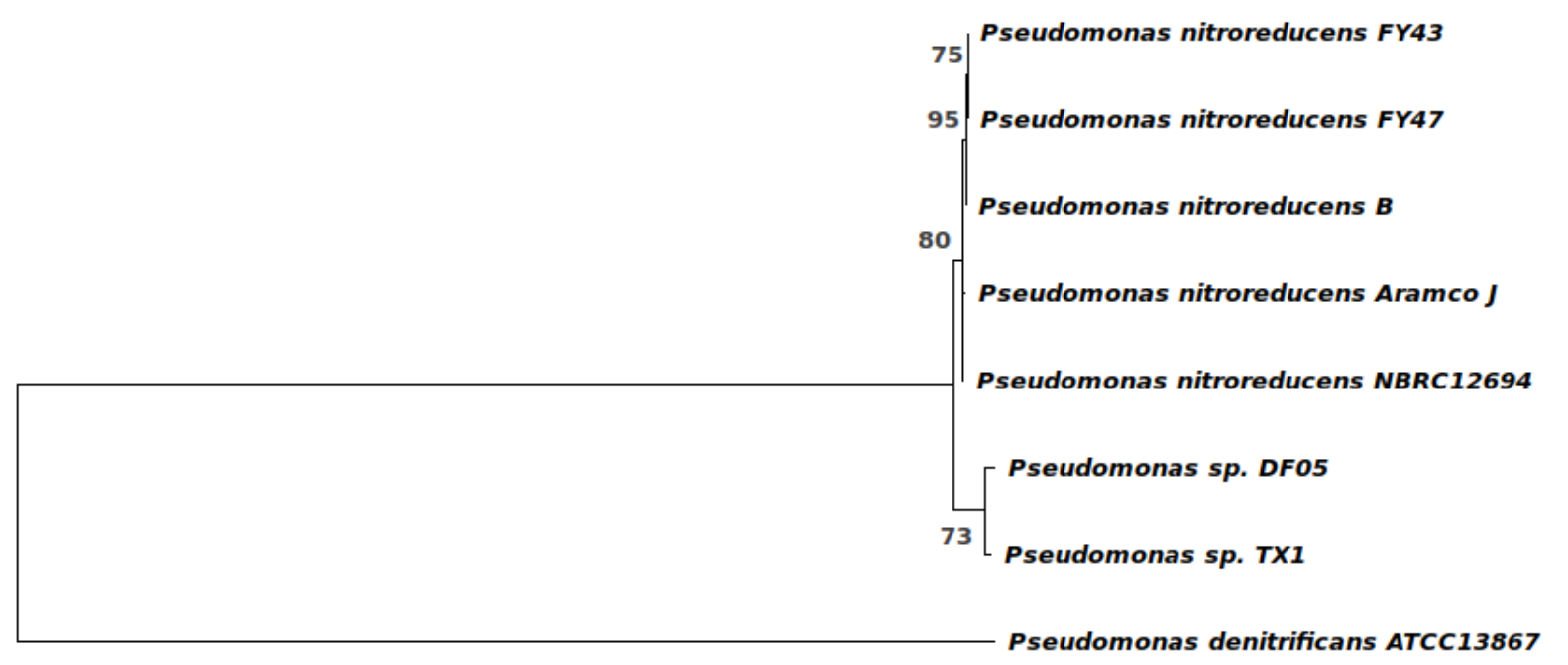

Figure 4. Phylogenetic tree of 8 Pseudomonas strains based on $r p o \mathrm{D}$ gene sequences.

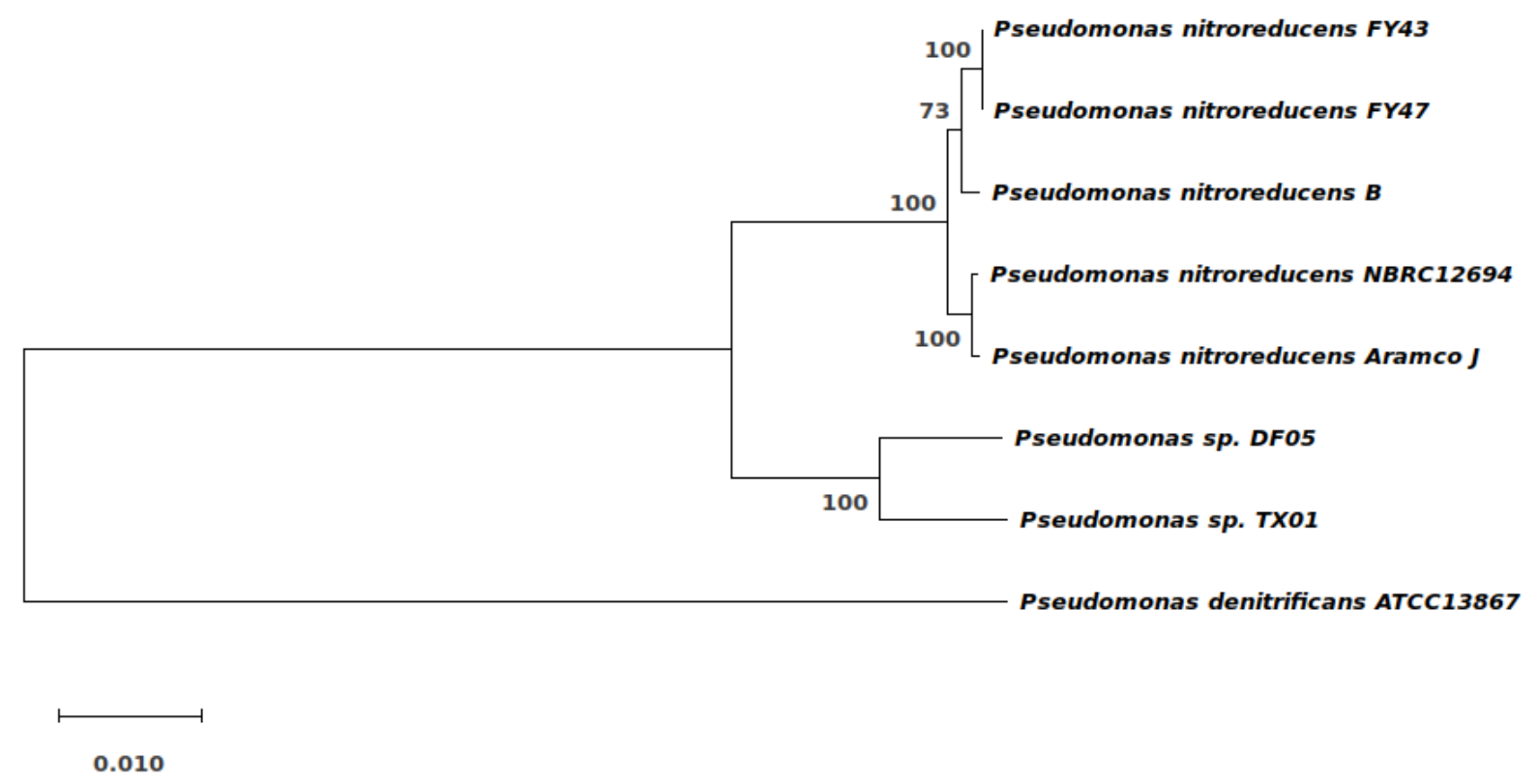

Figure 5. Phylogenetic tree of 8 Psendomonas strains inferred by MLSA. 


\begin{tabular}{llll} 
Reclassification $\quad$ of & \multicolumn{2}{c}{ Pseudomonas } \\
nitroreducens species & based on \\
phylogenomic analysis & &
\end{tabular}

To further confirm the identity of Pseudomonas nitroreducens strains FY43 and FY47, as well as Pseudomonas sp. strains DF05 and TX01, genomic analysis, including (i) ANIb, (ii) analysis based on MLSA, and (iii) GGDC, were utilized.

\section{Average nucleotide identity based on BLAST (ANIb) analysis}

The strains compared are classified as different species if the ANIb value is below $95 \%$, whereas above $95 \%$ indicates that there are of the same species (Garrido-Sanz et al., 2016). Pseudomonas nitroreducens strains FY43 and FY47 have a similarity lower than 95\% with Pseudomonas sp. strains DF05 and TX01. The ANIb values for $P$. nitroreducens strains FY43 and FY47 were above 95\% when comparing with reference $P$. nitroreducens strains B, Aramco J, and NBRC 12694 (Figure 6). This depicted that Psendomonas sp. strains DF05 and TX01 belong to the same species but different from $P$. nitroreducens strains B, Aramco J, NBRC 12694, FY43, and FY47.

ANIb matrix was generated based on Pseudomonas nitroreducens strain B, Aramco J, NBRC 12694, FY43, and FY47, Pseudomonas sp. strains DF05 and TX01, as well as Pseudomonas denitrificans strain ATCC13867 (highest similarity with Pseudomonas sp. strains DF05 and TX01 in accordance to results from BLASTN). $P$. nitroreducens strains B, Aramco J, NBRC 12694, FY43, and FY47 exhibit a pairwise ANIb of more than $95 \%$, clustering them into the same species (Figure 7). Pseudomonas sp. strains DF05 and TX01 showed low ANIb value $(<95 \%)$ to all Psendomonas sp., including P. nitroreducens strains B, Aramco J,
NBRC 12694, FY43, FY47, and Pseudomonas denitrificans strain ATCC13867, indicating that Pseudomonas sp. strains DF05 and TX01 do not belong to Pseudomonas nitroreducens or Pseudomonas denitrificans.

\section{Genomic analysis based on MLSA}

The calculations based on MLSA analysis were carried out to provide a better resolution for species delineation. Strain clustering in MLSA was maintained at a cut-off of 97\% (Gomila et al., 2017). In this study, the MLSA value of $P$. nitroreducens strains FY43 and FY47 was more than 97\%, whereas Pseudomonas sp. strains DF05 and TX01 were lower than 97\% (Figure 8). Moreover, the MLSA value of $P$. nitroreducens strains FY43 and FY47 in comparison with reference $P$. nitroreducens strains B, Aramco J, and NBRC 12694 was more than $97 \%$ (Figure 8 ). This confirmed that Pseudomonas sp. strains DF05 and TX01 do not belong to Pseudomonas nitroreducens.

\section{Genome-to-genome distance (GGDC) analysis}

The GGDC similarities were calculated between pairwise genomic comparisons to clarify phylogenetic assignments to species. The GGDC values utilize a specific distance formula, where the similarity of species is cut-off at $70 \%$ (Gomila et al., 2015). When comparing Pseudomonas nitroreducens strains FY43 and FY47 to reference $P$. nitroreducens strains $\mathrm{B}$, Aramco J, and NBRC 12694, we found that their GGDC values were more than 70\% (Figure 9). Similar to ANIb and MLSA analysis, Pseudomonas sp. strains DF05 and TX01 recorded less than 70\% GGDC. This result further confirmed that Pseudomonas sp. strains DF05 and TX01 may not be correctly assigned. 


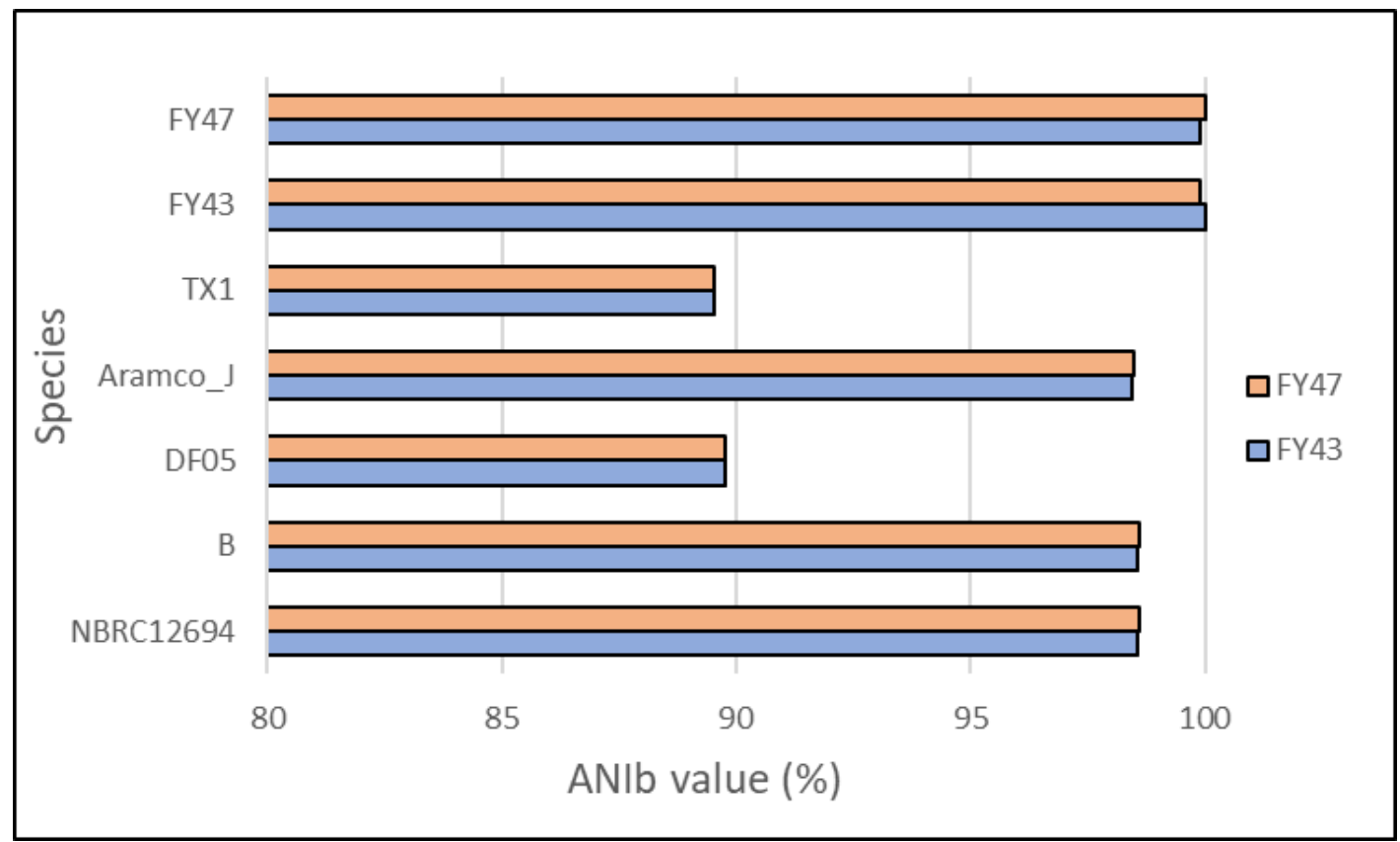

Figure 6. Genomic analysis of Pseudomonas sp. using ANIb analysis.

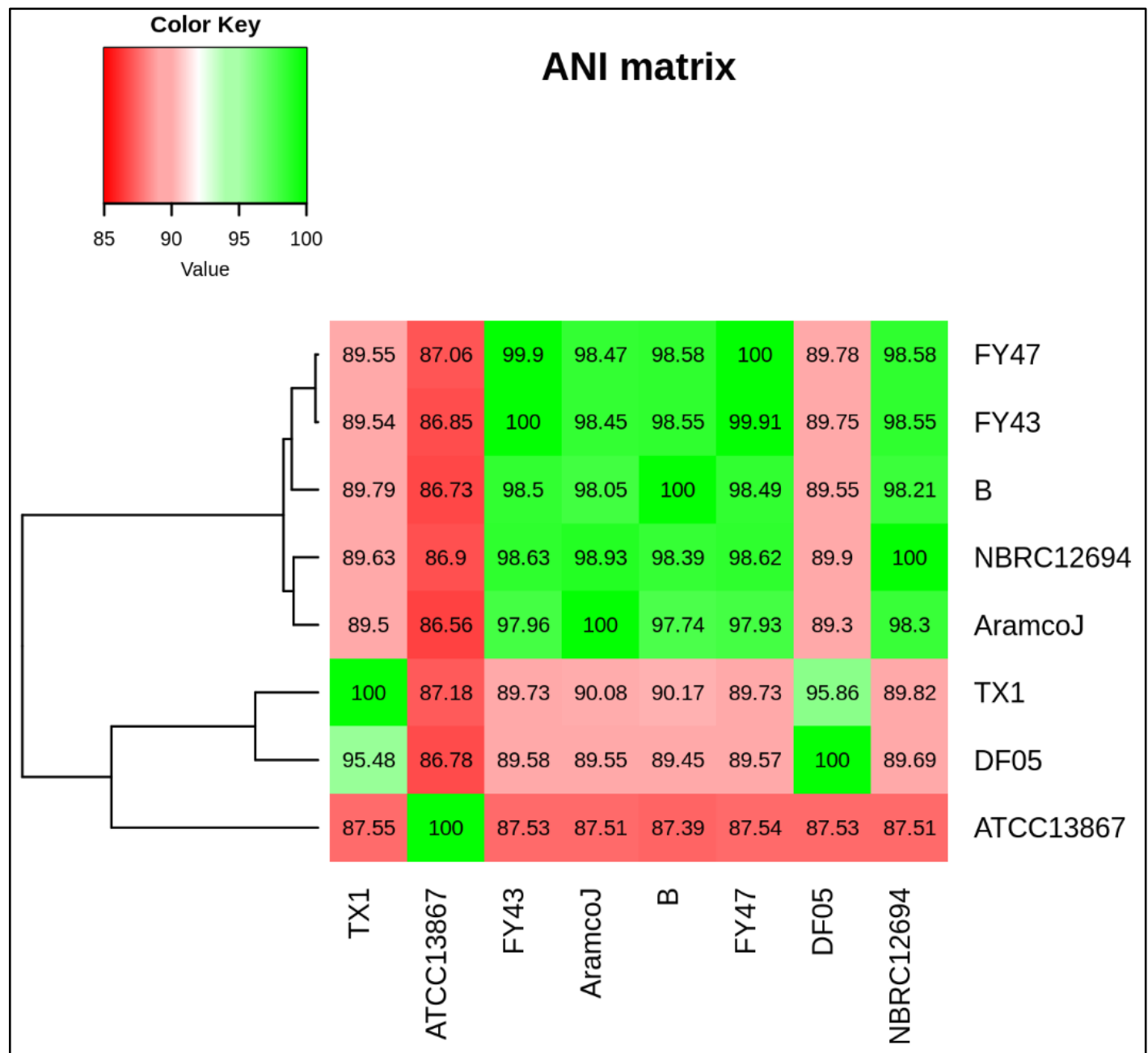

Figure 7. The ANIb matrix of 8 Psendomonas genomes from P. nitroreducens strains FY43, FY47, B, NBRC12694, Aramco J, Pseudomonas sp. strains DF05, TX01, and Pseudomonas denitrificans strain ATCC13867. 


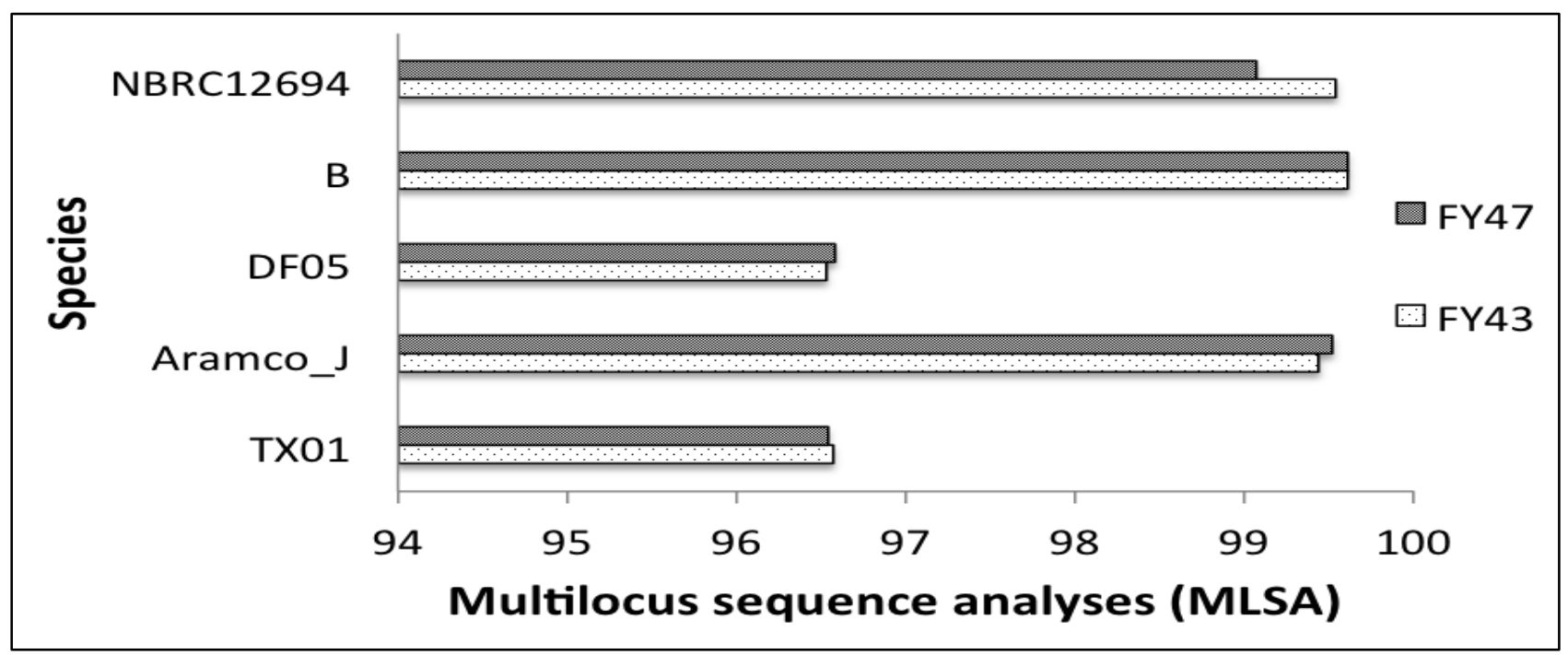

Figure 8. Genomic analysis of Pseudomonas sp. using MLSA analysis.

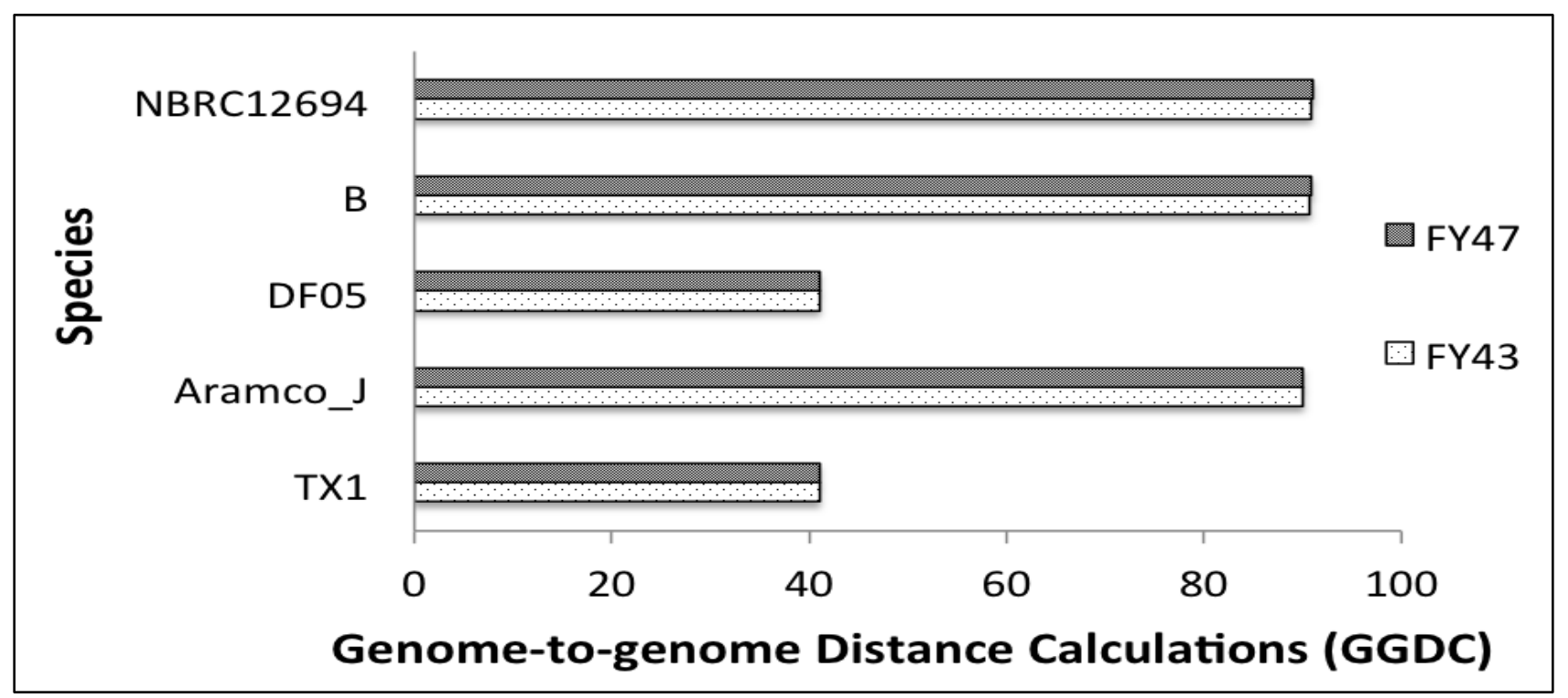

Figure 9. Genomic analysis of Pseudomonas sp. using GGDC analysis.

\section{CONCLUSION}

In this study, the genome of Pseudomonas nitroreducens stains FY43 and FY47 were extracted and sequenced using NGS approach. Our results indicated that $\mathrm{ABySS}$ assembly software can generate larger genome size, higher N50 values, and lower contig numbers compared to SPAdes and Velvet assemblers. The phylogenetic trees (16S rRNA, gyrB, rpoB, rpoD and MLSA) and phylogenomic analysis (ANIb, MLSA and GGDC method) confirmed that $P$. nitroreducens strains FY43 and FY47 are Pseudomonas nitroreducens.
Pseudomonas sp. strains DF05 and TX01, however, was not classified as the same species as $P$. nitroreducens strains B, Aramco J, NBRC 12694, FY43, and FY47. Therefore, the species delineation of Pseudomonas sp. strains DF05 and TX01 should be revised.

\section{ACKNOWLEDGEMENTS}

This study was supported by the Universiti Malaya grant, BKP078-2016 and RU006-2017. 


\section{CONFLICT OF INTEREST}

The authors have declared that no conflict of interests exists.

\section{REFERENCES}

Bankevich, A., Nurk, S., Antipov, D., Gurevich, A.A., Dvorkin, M., Kulikov, A. S., Lesin, V. M., Nikolenko, S. I., Pham, S., Prjibelski, A. D., Pyshkin, A. V., Sirotkin, A. V., Vyahhi, N., Tesler, G., Alekseyev, M. A., \& Pevzner, P. A. 2012. SPAdes: A new genome assembly algorithm and its applications to single-cell sequencing. Journal of Computational Biology 19: 455-477.

Chain, P. S. G., Grafham, D. V., Fulton, R. S., Fitzgerald, M. G., Hostetler, J., Muzny, D., Ali, J., Birren, B., Bruce. D. C., Buhay, C., Cole, J. R., Ding, Y., Dugan, S., Field, D., Garrity, G. M., Gibbs, R., Graves, T., Han, C. S., Harrison, S. H., Highlander, S., Hugenholtz, P., Khouri, H. M., Kodira, C. D., Kolker, E., Kyrpides, N. C., Lang, D., Lapidus, A., Malfatti, S. A., Markowitz, V., Metha, T., Nelson, K. E., Parkhill, J., Pitluck, S., Qin, X., Read, T. D., Schmutz, J., Sozhamannan, S., Sterk, P., Strausberg, R. L., Sutton, G., Thomson, N. R., Tiedje, J. M., Weinstock, G., Wollam, A., Consortium, G. S. C. H. M. P. J., \& Detter, J. C. 2009. Genomics. Genome project standards in a new era of sequencing. Science 326(5950): 236-237.

Chan, J. Z., Halachev, M. R., Loman, N. J., Constantinidou, C., \& Pallen, M. J. 2012. Defining bacterial species in the genomic era: insights from the genus Acinetobacter. BMC Microbiology 12: 302 .

Colston, S. M., Fullmer, M. S., Beka, L., Lamy, B., Gogarten, J. P., \& Graf, J. 2014. Bioinformatic genome comparisons for taxonomic and phylogenetic assignments using Aeromonas as a test case. $m$ Bio 5(6): $\mathrm{e} 02136$.

Euzéby, J. P. 1997. List of bacterial names with standing in nomenclature: A folder available on the internet. International Journal of Systematic and Evolutionary Microbiology 47: 590-592.

Garrido-Sanz, D., Arrebola, E., Martínez-Granero, F., GarcíaMéndez, S., Muriel, C., Blanco-Romero, E., Martín, M., Rivilla, R., \& Redondo-Nieto, M. 2017. Classification of isolates from the Pseudomonas fluorescens complex into phylogenomic groups based in group-specific markers. Frontiers in Microbiology 8: 413.

Garrido-Sanz, D., Meier-Kolthoff, J. P., Göker, M., Martín, M., Rivilla, R., \& Redondo-Nieto, M. 2016. Genomic and genetic diversity within the Pseudomonas fluorescens Complex. PLOS ONE 11: e0153733.

Glaeser, S. P. \& Kämpfer, P. 2015. Multilocus sequence analysis (MLSA) in prokaryotic taxonomy. Systematic and Applied Microbiology 38(4): 237-245.

Gomila, M., Busquets, A., Mulet, M., García-Valdés, E., \& Lalucat, J. 2017. Clarification of taxonomic status within the Pseudomonas syringae species group based on a phylogenomic analysis. Frontiers in Microbiology 8: 2422.

Gomila, M., Peña, A., Mulet, M., Lalucat, J., \& García-Valdés, E. 2015. Phylogenomics and systematics in Pseudomonas. Frontiers in Microbiology 18: 214.

Goris, J., Konstantinidis, K. T., Klappenbach, J. A., Coenye, T., Vandamme, P. \& Tiedje, J. M. 2007. DNA-DNA hybridization values and their relationship to whole- genome sequence similarities. International Journal of Systematic and Evolutionary Microbiology 57(1): 81-91.

Jun, S., Wassenaar, T. M., Nookaew, I., Hauser, L., Wanchai, V., Land, M., Timm, C. M., Lu, T. S., Schadt, C. W., Doktycz, M. J., Pelletier, D. A., \& Ussery, D. W. 2016. Diversity of Pseudomonas genomes, including populus-associated isolates, as revealed by comparative genome analysis. Applied and Environmental Microbiology 82: 375-383.

Konstantinidis, K. T., Ramette, A., \& Tiedje, J. M. 2006. Toward a more robust assessment of intraspecies diversity, using fewer genetic markers. Applied and Environmental Microbiology 72: 7286-7293.

Lalucat, J., Mulet, M., Gomila, M., \& García-Valdés, E. 2020. Genomics in bacterial taxonomy: Impact on the genus Pseudomonas. Genes (Basel) 11(2): 139.

Looi, F. Y. 2016. Isolation and characterization of glyphosate degrading bacteria from oil palm plantation soil sample. Bachelor theses, Taylor's University, Subang Jaya

Maiden, M. C., Bygraves, J. A., Feil, E., Morelli, G., Russel, J. E., Urwin, R., Zhang, Q., Zhou, J., Zurth, K., Caugant, D. A., Feavers, I. M., Achtman, M., \& Spratt, B. G. 1998. Multilocus sequence typing: a portable approach to the identification of clones within populations of pathogenic microorganisms. Proceedings of the National Academy of Sciences of the United States of America 95: 3140-3145.

Meier-Kolthoff, J. P., Auch, A.F., Klenk, H. P., \& Göker, M. 2013. Genome sequence-based species delimitation with confidence intervals and improved distance functions. $B M C$ Bioinformatics 14: 60.

Molina-Mora, J. A., Campos-Sánchez, R., Rodríguez, C., Shi, L., \& García, F. 2020. High quality 3C de novo assembly and annotation of a multidrug resistant ST-111 Pseudomonas aeruginosa genome: Benchmark of hybrid and non-hybrid assemblers. Scientific Report 10: 1392.

Mulet, M., Bennasar, A., Lalucat, J., \& García-Valdés, E. 2009. An rpoD-based PCR procedure for the identification of Pseudomonas species and for their detection in environmental samples. Molecular and Cellular Probes 23: 140-147.

Mulet, M., García-Valdés, E., \& Lalucat J. 2013. Phylogenetic affiliation of Pseudomonas putida biovar A and B strains. Research in Microbiology 164: 351-359.

Mulet, M., Lalucat, J., \& García-Valdés, E. 2010. DNA sequencebased analysis of the Pseudomonas species. Environmental Microbiology 12: 1513-1530.

Novik, G., Savich, V., \& Kiseleva, E. 2015. An insight into beneficial Pseudomonas bacteria. Microbiology in Agriculture and Human Health 10.5772/60502.

Parte, A. C. 2014. LPSN-List of prokaryotic names with standing in nomenclature. Nucleic Acids Research 42(D1): D613-D616.

Richter, M. \& Rosselló-Móra, R. 2009. Shifting the genomic gold standard for the prokaryotic species definition. Proceedings of the National Academy of Sciences of the United States of America 106: 19126-19131.

Simpson, J. T., Wong, K., Jackman, S. D., Schein, J. E., Jones, S. J., \& Birol, I. 2009. ABySS: a parallel assembler for short read sequence data. Genome Research 19(6): 1117-1123.

Stanier, Y., Pallerom, N. J., \& Doudoroff, M. 1966. The aerobic pseudomonads: a taxonomic study. Journal of General Microbiology 43: 159-271.

Tan, X. L., Othman, R. Y., \& Teo, C. H. 2020. Isolation and functional characterization of 5-enolpyruvylshikimate 3-phosphate synthase gene from glyphosate-tolerant Pseudomonas nitroreducens strains FY43 and FY47. 3 Biotech 10: 183.

Tarazona, E., Lucena, T., Arahal, D. R., Macián, M. C., Ruvira, M.A., \& Pujalte, M. J. 2014. Multilocus sequence analysis of putative Vibrio mediterranei strains and description of Vibrio 
thalassae sp. nov. Systematic and Applied Microbiology 37: 320 328.

Thakur, S., Weir, B. S., \& Guttman, D. S. 2016. Phytopathogen genome announcement: Draft genome sequences of 62 Pseudomonas syringae type and pathotype strains. Molecular Plant-Microbe Interactions: MPMI 29(4): 243-246.

Thompson, J. D., Gibson, T. J., Plewniak, F., Jeanmougin, F., \& Higgins, D. G. 1997. The CLUSTAL_X windows interface: flexible strategies for multiple sequence alignment aided by quality analysis tools. Nucleic Acids Research 25: 4876-4882.

Yumoto, I., Kusano, T., Shingyo, T., Nodasaka, Y., Matsuyama, H., \& Okuyama, H. 2001. Assignment of Pseudomonas sp. strain E-3 to Pseudomonas psychrophila sp. nov., a new facultatively psychrophilic bacterium. Extremophiles 5(5): 343-349.

Zerbino, D. \& Birney, E. 2008. Velvet: algorithms for de novo short read assembly using de Bruijn graphs. Genome Research 18(5): 821-829. 